\title{
Synthesis of MOF-5@HMSS composite material and performance evaluation of adsorption desulfurization in oil ${ }^{*}$
}

\author{
Xue-Ying Jia, Zheng-Tai Zhang, Li-Jun Zhu ${ }^{\dagger}$ and Dao-Hong Xia \\ State Key Laboratory of Heavy Oil Processing, \\ China University of Petroleum, \\ Qingdao, 266580, China. \\ E-mail: zhulijun@upc.edu.cn
}

\begin{abstract}
Micro-mesoporous composite material MOF-5@HMSS was synthesized by "in-situ" synthesis during the synthesis of MOF-5. The synthesized composite materials were characterized by XRD and SEM. The adsorptive capacity of thiophene compounds in simulated fuel with MOF-5@HMSS materials was investigated using static adsorption method. The composite materials showed higher adsorption efficiency for thiophene in comparison with MOF-5 and HMSS.
\end{abstract}

Keywords: MOF-5; HMSS; Micro-Mesoporous Composite; In-Situ..

\section{Introduction}

Considering the requirements for environmental protection, deep desulfurization is essential for petrochemical industry. The adsorption process is recognized as one of the most promising methods for desulfurization is due to the process conditions of low temperature and low pressure, and low cost inexpensive[1]. With all its advantages, the porous materials are by no means without their limitations to desulfurization efficiency.

Metal-organic frameworks (MOFs) have aroused much attention for adsorption due to the huge variety of structures, large surface areas, ultrahigh porosity and high functionality. MOF-5 is one of the most prominent representatives of these 3D porous MOFs. However, MOF-5 is mostly microporous and skeletons of MOF-5 are often interpenetrate during the crystallization process and tend to collapse upon sulfur compounds removal[2]. In addition, ordered mesoporous silica materials as highly efficient adsorbents have been extensively applied on the field of adsorption. Owing to the thermal

\footnotetext{
* Work partially supported by National Natural Science Foundation of China and the Fundamental Research Funds for the Central Universities.
} 
stability, high porosity, hollow space of HMSS, it can be adopted as a support for MOFs to form micro-mesoporous composite for deep desulfurization[3].

In this work, we synthesized MOF-5@HMSS micro-mesoporous composite material for enhancing the adsorption capacity by in-situ hydrothermal methodology. The X-ray diffraction (XRD) X-ray was used to investigate the frameworks of micro-mesoporous composite materials. And the adsorptive desulfurization properties of MOF@HMSS for the thiophene in simulated oil were measured.

\section{Experimental}

\subsection{Synthesis of HMSS}

The synthesis of hollow mesoporous silica spheres (HMSS) was implemented with according to a previous reported procedure[4]. The method includes the following steps: (1) preparation of solid $\mathrm{SiO}_{2}$ spheres $\left(\mathrm{sSiO}_{2}\right)$ by a modified Stober method. (2) Synthesis of HMSS by selective etching.

\subsection{Synthesis of MOF-5@HMSS}

The MOF-5 was prepared following literature procedure[5]. MOF-5@HMSS composites were prepared by "in-situ" synthesis shown as below. Firstly, asprepared HMSS (2g) was added into the solution of $\mathrm{Zn}\left(\mathrm{NO}_{3}\right)_{2} \bullet 6 \mathrm{H}_{2} \mathrm{O}(0.178 \mathrm{~g})$ dissolved in DMF $(50 \mathrm{~mL})$ and was sonicated for $5 \mathrm{~min}$. Secondly, the 1,4benzenedicarboxylic acid $\left(\mathrm{H}_{2} \mathrm{DBC}, 0.033 \mathrm{~g}\right)$ was added into the mixture. After sonication for another $5 \mathrm{~min}$, the resulting suspension heated up to $130{ }^{\circ} \mathrm{C}$ for $8 \mathrm{~h}$ in a Teflon-Steel autoclave. And the reaction products were cooled down to room temperature and filtered off. The remaining solid was washed with DMF (50 mL×3) and dip in $\mathrm{CH}_{2} \mathrm{Cl}_{2}$ for three days to exchange the DMF in pore.

\subsection{Characterization}

The XRD measurements were recorded by Panalytical X'Pert PRO MPD diffract meter. SEM images were recorded with a Hitachi S-4800 microscope.

\subsection{Adsorption desulfurization experiments}

All samples were activated undervacuum at $150{ }^{\circ} \mathrm{C}$ for $8 \mathrm{~h}$ before adsorption desulfurization experiments. And the simulated oil was obtained by dissolving thiophene compounds $(500 \mu \mathrm{g} / \mathrm{g})$ in $\mathrm{n}$-heptane. The desulfurization capacities of samples were evaluated by the static adsorption method at $30 \circ \mathrm{C}$ (adsorbent-oil ratio=1:20). The S-content of supernatant was analyzed by an ANTEK9000 element analyzer. And desulfurization capacity of absorbent was calculated by 
the following formula: $\mathrm{Q}_{t}=\rho \mathrm{V}\left(\mathrm{C}_{0}-\mathrm{C}_{\mathrm{t}}\right) / 1000 \mathrm{M}$, where $\mathrm{Q}_{\mathrm{t}}$ is the adsorption capacity of adsorbent $(\mathrm{mg} / \mathrm{g}), \rho$ is the density of the simulated fuel at room temperature $\left(\mathrm{g} / \mathrm{cm}^{3}\right), \mathrm{V}$ is the volume of the simulated fuel $(\mathrm{mL}), \mathrm{M}$ is the mass of the adsorbent $(\mathrm{g}), \mathrm{C}_{0}$ is the initial S-content of the simulated fuel $(\mu \mathrm{g} / \mathrm{g})$, and $\mathrm{C}_{\mathrm{t}}$ is the $\mathrm{S}$-content of the simulated fuel $(\mu \mathrm{g} / \mathrm{g})$ at any time $\mathrm{t}(\mathrm{h})$.

\section{Results and discussion}

\subsection{XRD characterization}

The Figure 1(A) shows small angle XRD patterns of pure HMSS and MOF$5 @$ HMSS. The characteristic HMSS signal corresponding to (100) plane was preserved, proving that the structure of HMSS remained intact after compositing with MOF-5. The wide angle XRD patterns of samples are compared in Figure 1(B). The characteristic peaks of MOF-5@HMSS appear at the $2 \theta$ values of $6.8^{\circ}$, $9.7^{\circ}, 13.4^{\circ}$ and $15.7^{\circ}$, indicating the formation of MOF-5.
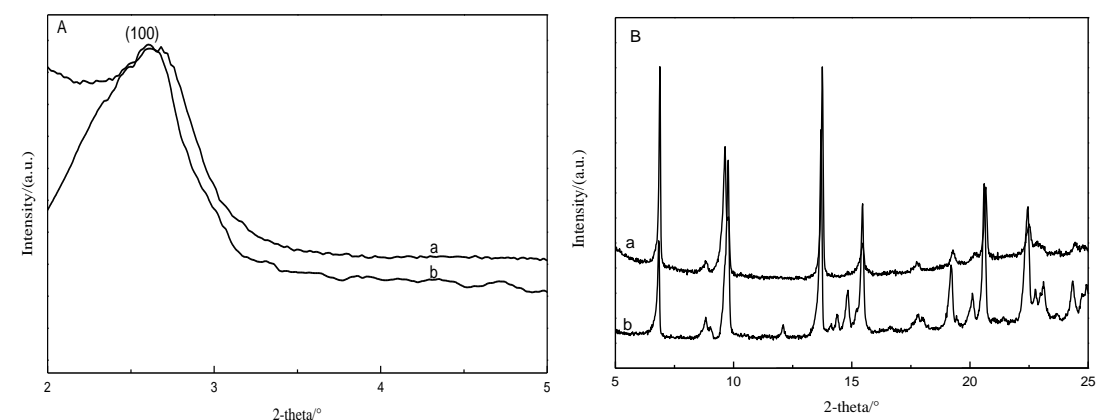

Fig. 1. (A) Small angle XRD pattern of (a)HMSS, (b) MOF-5@HMSS and (B)wide angle XRD pattern of (a) MOF-5@HMSS, (b) MOF-5.

\subsection{Scanning electron microscopy (SEM)}

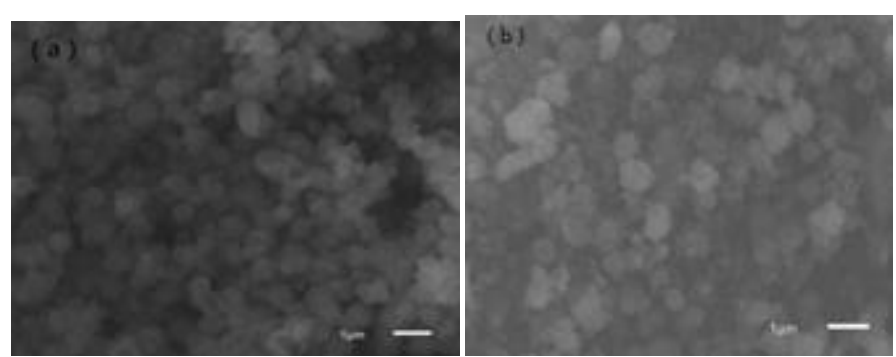

Fig. 2. SEM images of (a) HMSS and (b)MOF-5@HMSS.

The SEM micrographs of HMSS and MOF-5@HMSS are plotted in Fig.2. The SEM images indicate that the pure HMSS is hollow spherical with uniform size $\sim 400-500 \mathrm{~nm}$. In addition, it observed that HMSS has partial agglomeration 
phenomenon. The micrograph of MOF@HMSS shows that the surface of composite is rougher than pure HMSS and the size of particle increases.

\subsection{Adsorption desulfurization experiments}

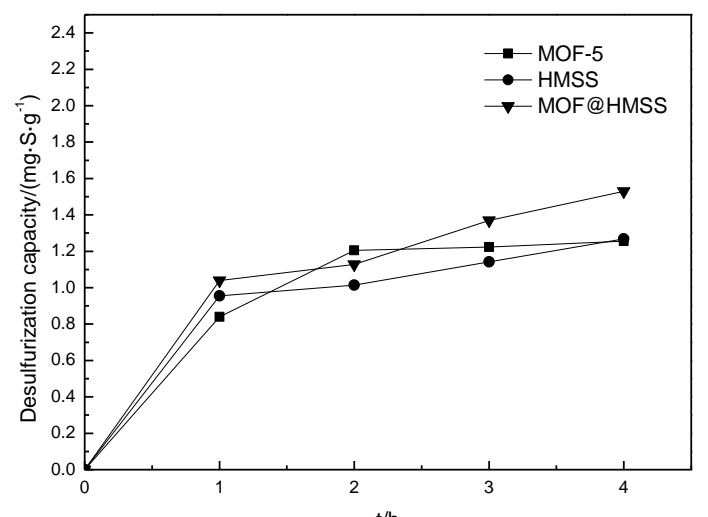

Fig. 3. The Adsorption desulfurization curves of MOF-5, HMSS, or MOF-5@HMSS

The Figure 3 shows the desulfurization results of thiophene in simulated fuel over MOF-5, HMSS, and MOF-5@HMSS. As the adsorption time is prolonged to $4 \mathrm{~h}$, the curves tend to increase laxly, indicating that the adsorption approximately reaches saturation. And the MOF-5@HMSS exhibited higher desulfurization capacity in comparison with MOF-5 and HMSS. Among in MOF-5@HMSS, micropores provide selectivity porous for thiophenic compounds, while mesopores supply easier access to the adsorption active sites.

\section{Conclusions}

In order to deep desulfurization of fuel oil, MOF-5@HMSS micro-mesoporous composite material was prepared by in-situ hydrothermal synthesis. And the MOF-5 is produced on HMSS by XRD and SEM. The micro-mesoporous composite materials show higher adsorption capacity than HMSS because of the particular pore structure. Choosing HMSS as a support of MOFs provide a new train of thought on the creation of excellent architectures and highly efficient micro-mesoporous composite materials.

\section{References}

1. B. Van de Voorde and M.Hezinova, Adsorptive desulfurization with CPO27/MOF-74:an experimental and computational investigation.Phys. Chem. Chem. Phys.17,10759(2015). 
2. A. Kondo and S. Takanashi, New insight into mesoporous silica for nano metal-organic framework. J. Colloid Interf. Sci. 384, 110(2012).

3. S. Sorribas and B. Zornoza, Ordered mesoporous silica-(ZIF-8) core-shell spheres. Chem. Commun. 48, 9388(2012).

4. X. Fang and C. Chen, A cationic surfactant assisted selective etching strategy to hollow mesoporous silica spheres. Nanoscale3, 1632(2011).

5. U. Mueller and M. Schubert, Metal-organic frameworks-prospective industrial applications. J. Mater. Chem.16, 626(2006). 